Article

\title{
Design is how we change the world! Can we do it in socially, environmentally and economically acceptable ways? Synthesizing design tools for this utopian concept
}

\author{
Kumaraswamy Ponnambalam 1,", Abbas Seifi ${ }^{2}$ and Jamshid Mousavi ${ }^{3}$ \\ 1 Department of Systems Design Engineering, University of Waterloo, Waterloo, Ontario, Canada, N2L 3G1; \\ ponnu@uwaterloo.ca \\ 2 Department of Industrial Engineering, Amirkabir University of Technology (Tehran Polytechnic), Tehran, \\ 15875-4413, Iran; aseifi@aut.ac.ir \\ 3 School of Petroleum, Civil and Mining Engineering, Amirkabir University of Technology (Tehran \\ Polytechnic), Tehran, 15875-4413, Iran; jmosavi@aut.ac.ir \\ * Correspondence: ponnu@uwaterloo.ca
}

\begin{abstract}
Since the beginning, humans advanced their civilization by making better tools to improve their lives. Tools and products for better living were designed considering engineering (manufacturing) issues and cost (time and money as predominant criteria). It has become clear that not considering environment and society, both at local/global levels, has now become a major impediment affecting living conditions of large parts of earth and society. Design methodologies should lead to creative solutions considering engineering and economics for practicality but also environmental and social constraints for longevity. We propose a comprehensive design methodology based on multidisciplinary design for including the knowledge of humanities, and science and engineering and allowing for experts from these areas to provide various necessary inputs. For example, experts in humanities are expected to interact with stakeholders to evaluate their value systems to provide guidance for the design. The methodology that we synthesize is new and combines (i) Societal level impacts at all scales, (ii) Environmental impacts and (iii) Engineering design with economic impacts, including the consideration of uncertainties. The proposed SocialEnvironmental-Economical-Engineering-based-design Framework (SEEEF) can utilize tools such as circular design, donut economics, design based on environmental life cycle analysis, among others. SEEEF is quantity based and provide steps for evaluating any project or product in an objective manner and will help train engineers in design for sustainability and provide non-engineers a significant role in design and to increase their understanding of the hard constraints of engineering. Ultimately, SEEEF allows society to take an informed decision considering short/long term and local/global impacts much of which are affected by uncertainty.
\end{abstract}

Keywords: design for society, design for sustainability, design under uncertainty, circular design, donut economics, life cycle analysis

\section{Introduction}

Economics and Engineering based designs go hand in hand especially since the industrial era. Industrial production meant that a societal demand was expected for the product so some aspects of the Society was considered. Local Environmental issues were most likely considered either for practicality (earlier days) and by societal regulations (later days). Until recently, global impacts were rarely considered and the ability to even consider various temporal and spatial impacts on local/global effects are still in development [1]. However, we know now almost all products have 
both local and global impacts on both environment and society, even if small at a given time/space coordinate but over some length of time the cumulative impacts are significant. Considering product design including engineering, economics, environmental and social constraints may appear daunting (here, unless we say specifically, local and global, and short and long terms are always considered in the design process), and at these scales uncertainty is a major component and is included. However, sufficient understandings of how one might consider individually these constraints in a design, even under uncertainty, are available but no design framework exist that combines all these objectives jointly. One may wonder that, even if we have such a design framework, named as the SocialEnvironmental-Economical-Engineering-based-design Framework (SEEEF), see Figure 1, given the national divisions and political philosophies, will it be practical? Available methods include: circular design [2] product design using large amount of data now available [3], and (environmental) life cycle design [4], bioinspired sustainable product design [5] and sustainable design could that could induce change in consumer behavoiur [6]; in addition, trade and bi-lateral and international agreements control most products; our aim in this paper is to use all these existing ideas to develop a framework that is implementable at various levels considering both local and global constraints. Recently, Amsterdam city has agreed to a progressive design procedure called the Donut economics [7-8] that considers the same issues we will tackle. The difference is that we provide synthesis of existing design methods with new metrics where required to consider local/global and short/long term impacts and can be applied in practice to achieve what is analyzed in [8]. It is also noted that both Environmental and Engineering modules depend on both fundamental and applied science and hence there no separate module for science. In other words, each of these modules have been used alone as a decision tool in the past and SEEEF provides a method for combining all four views at the levels required to be of use in practice.

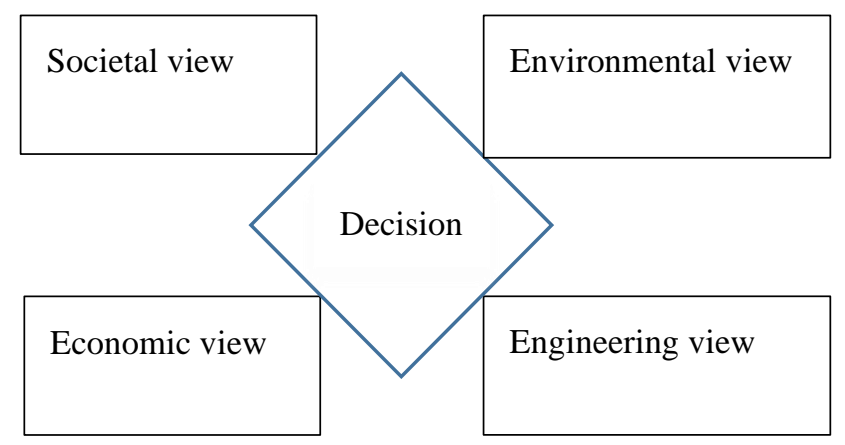

Figure 1: SEEEF Diagram

Motivational example: Recently Joe Biden, a candidate for presidency in the USA announced a $\$ 2$ Trillion investment to bring on more renewable energy in the country [9]; this is a major design problem affected by all four views considered in SEEEF and we will use this as an example (named REPUSA) to demonstrate the design process and various individual methods. There are conflicting opinions on this energy project including the biggest problem being of societal acceptance. It is not hard to imagine the not-in-my-backyard (NIMBY) phenomenon as one of the societal problem, in addition to problems such as uncertainty in demand and supply, land costs, environmental issues, trade and other policy issues, and any design must meet constraints of economy and engineering. In today's societal situation, where a large number of people are unemployed, even if costs of materials for this solution and imported labour to install is much cheaper from other countries under a trade agreement, the solution of simply importing may be unacceptable for a large part of the local population. Our aim in this paper is to provide details of SEEEF synthesizing various design methodologies considering not just engineering and economics, with just slight adjustments considering environment and society, but to provide a method for holistic design considering all 
these four views from the beginning. A project may include just a single product and such a situation is more common to an individual company (iPhone of Apple is a good example) or many "products" which is likely the mandate of governmental level organizations (REPUSA). SEEEF includes methods for products and projects and can utilize donut economics which is primarily an evaluation tool and more comprehensive than circular design which is product specific and does not include societal influences.

\section{Designing at societal level: Feedbacks and scale challenges}

Designing at societal level means that one has to consider impacts on humans at local, regional, national and global levels as well as considering both short and long terms due to intergenerational challenges. On the other hand, it is obvious that considering societal impacts cannot be done without interacting with other views and SEEEF will address this.

Design of systems considers the system as a whole and society is an example of a system to be studied in the society view module of SEEEF. Identifying all components of a system is a challenging activity but has been made easier with large amount of knowledge and data available even in electronically readable forms today (for example, as necessary for environmental life cycle analysis LCA [10] by one of the early proponent-the Society for environmental toxicology and chemistry commonly known as SETAC). Each individual and interacting component of the system, whether it may be civil, electrical, mechanical, computational, biological, societal, among others, is simply represented by its functionality and its interface. While the detailed design of individual components may be the responsibility of each field, integrating these diverse components such that they work together effectively as a whole is our design objective.

Design is a creative problem solving exercise; identifying stakeholders, their objectives, their criteria and corresponding measures form the very first steps. While designing for society the objectives could be separated by short and long term, and local and global and feedback between these differing scales. For example, in REPUSA, the short term objective could be renewable energy (for simplicity we will consider just photovoltaic solar energy only here) to meet current local demand at a certain percentage of total local demand but for long term this percentage can be changing to a higher value (the optimal value itself will be result of the application of SEEEF). In addition to this objective, the REPUSA may be forced by international agreements to have a stepwise long term plan for the entire USA, thus changing local installment capacities as part of long term strategy. It is also noted that an international agreement on say, greenhouse gases (GHG) limitations, specific for the USA can be converted to equivalent renewable energy (assuming all of the targets are provided solely by energy sector which, of course, is a simplification for explanation purposes and can be extended to sector by sector targets). However, at the societal level meeting energy targets by renewable energy is just one objective and can be measured in kilowatt per hour energy met. On the other hand, this energy-environment objective even if achieved may be completely unacceptable if we don't consider other societal goals such as maintaining an employment ratio, sufficient income meaningful for local conditions (as it may be necessary to reduce employment existing from other sources of energy locally produced), not degrading aesthetics (other environment related societal constraints; for example, how much local and global toxicity can be allowed and for how long and what current and future remedial solutions have been identified all can be dictated by society to the environment view module), not degrading land use for other purposes like farming and forestry, no adverse health impacts, and equitable share of land resources for energy generation (for example, the province of Ontario in Canada, during the McGuinty government, received public input regarding the extension of what is called the Microfit program that supported increased solar energy installations; the first author suggested, possibly among others, a model where local body of people should have significant share of the renewable energy development and this scheme became a bigger success and with much less NIMBY problem and increased significantly renewable energy installations in Ontario). Unless an objective such as equality or equity and the stakeholders affected has been identified, it is not possible to find a solution that required policy changes at the various governing levels. 
In summary, the societal view module of SEEEF will accept inputs such as plans and policies and return merit scores for various objectives affecting all stakeholders. Societal module input include society's objectives (targets time series, allowing for progressive changes, for income, equality index, environmental values, and others; even aesthetics can be specified (for example, with stakeholder meetings targets of non-aesthetic parts of solutions can be restricted such as requiring no more than $10 \%$ of the area for solar panel layout, etc) and possible plans and solutions. SEEEF will pass these plans/policies/targets to other modules to get appropriate scores and sensitivity of these scores for chosen decisions and constraints. The scores will have short term and long term results corresponding to various local regions identified and global (sum of all local regions and beyond). Scores will be presented with at least two lower moments (means and (co)variances for each criterion) so that uncertainty is explicitly stated. It is also possible to include fuzzy logic analysis to consider uncertainties that are not available in a statistical sense. A decision screening analysis can be done using mutiobjective and multicriteria (even the same objective can produce muticriteria results, for example, REUSA demand satisfaction according to a constraint can produce employment results in income as well as equality levels, for example, the Gini index that measures equality) and a chosen selection method can be applied for choosing the decision (many methods exist such as AHP [11], conjoint analysis of choice [12], and so on, and are not provided in details here). In the Engineering module, described later, detailed parts of designs is being considered which, of course, is not needed at the higher level of decision making. The decision module where final decisions is made with tools as above is also a communication module between various other modules passing the required inputs to those modules and collecting the outputs of those modules to pass on to other modules.

\section{Design guided by environmental life cycle analysis}

Environmental life cycle analysis (as opposed to life cycle analysis in economics which is mainly concerned with useful machine life) can be used to design products considering their impacts from cradle-to-cradle. Products go through many transformative processes, namely, mining raw natural sources (from earth which is the cradle) to processing to manufacture to transportation to consumptive use and eventually disposed of to nature (cradle again). Moreover, at each of the intermediate steps effluents of the processes also go to nature and may require inputs such as land, water, air, and energy. The impacts of any product is measureable (whose accuracy is continuously improving since the 1990s) in terms of target environmental variables, for example, land area used, GHG emissions, toxicity to water and land, among others, and available from software such OpenLCA [13]. Any proposed design is evaluated in terms of environmental quality indices for the given design coming from the engineering module.

For the example of REPUSA, the constraints on land, GHG emissions and aquatic toxicity are some of the key environmental target variables. This could vary both locally and globally as well as can be different for various time periods. Taking the example of some rare metals used in photovoltaic systems, the target for next few years (the length itself can be a design variable) can be different. It is possible that with given targets more incentives exist either to design such that it can be recycled or replace some of the rare metals which are highly toxic over a longer period of time. Both circular product design [2] and sustainability based product design [4] consider these in detail. A difference between these and the proposed Environmental module in SEEEF is that we will include uncertainty in all our designs so output scores on various environmental variables include both means and (co)variances of impacts to take final decisions whose risk factor will be jointly available with expected scores. Also, another major difference is that the scores are expected as a function of time and location. For example, source pollution is given separately from transportation pollution (for example, if main transport is through ocean then it is separately provided compared to pollution from land transport) and pollution during consumption/disposal. This level of detail is necessary to bring more equity as pollution in a city has more impacts than on an unpopulated area; however, in a global sense cumulative impacts will be considered as pollution anywhere can result in problem everywhere, for example, the GHG emissions! 


\section{Design under uncertainty in cost, manufacturing and engineering}

This section covers the well-known highly interlaced Economic and Engineering modules. Because we would want to understand both local and global issues we will also discuss trade issues as trading makes it more efficient in an economic sense. The content in these modules have been in development for a long time and hence we provide only what is the minimum needed to understand SEEEF. Most of the development in civilization came from one major economic concept called the economy of scale which, in simple sense, translates to lower costs of products when produced in large quantities and hence increasing their availability to many. This concept cannot be implemented without applying engineering principles. For example, ancient humans figured out how to make mud pots (which lead to food that could be cooked with water, leading to a huge jump in living conditions due to easy availability of digestible food), and in the beginning only artisans were able to make these pots. However, as soon as the wheel was invented (an important start for engineering), artisans not only could make many pots in the same time, they were able to train others to create even a larger number of pots. A craft work migrated to be an engineering work (a similar pattern followed also in software that went from code crafted by a few to APIs that many can use producing innumerous number of Apps at hugely reduced cost). Hence the drop in the cost of a pot increased the possibility of more people acquiring it. The concept of economy of scale tied economics with engineering. The reason that so many products are being manufactured today in China is because the economy of scale provided by them due to the availability of a large number of cheap-skilled-disciplined labour is unmatched by any other today but the USA was in a similar situation after the second world war and it still has cheap farm labour and relatively inexpensive software engineers, both due to a large number of people who arrived from other countries. Between these two countries, this has led to the balance of trade favourable to China in consumer products manufactured in factories but it is favourable to the USA for products (services mostly) that depend on a large number of skilled labour (software engineers are a major group here) in the service industries.

The same economy of scale that made it possible for innumerous number of products affordable to a large population also produced a large side effect which is the pollution from waste. People could acquire a large number of products as they were available at a much cheaper price but a lot of them were not "necessary" products ending up as being unused or thrown as a waste. Therefore, the same scale of economy's major side effect is an important reason why we need the environmental module in SEEEF. These pollution costs were originally considered by economists as "external", as the pollution generally affected neither the producers nor the consumers (in the first instance). In order to make the polluter pay, laws had to be formulated (to be considered in the societal module), to discourage "unnecessary" buying and to discourage producing "non-recyclable" products. Local costs are simply fees like deposit fees but costs from global impacts are to be taken care of by international agreements leading to costs such as the carbon tax. Therefore, the economic module will be used to make decisions on costs (both of the local kind and of the global kind) and will be used in the engineering module. It is noted that import taxes are nationalistic way of making products more locally produced (so increase in local employment) and this must be traded off with other side effects such as local pollution and reduced consumption from higher costs on products, which is not necessarily bad for the environment.

A major input to engineering module is the short and long term demands and costs available from the economic module as well as their uncertainties. Uncertainties at the moment are considered available from economic module, however, those uncertainties themselves could be affected by solutions that are provided by the engineering module, hence, the idea that these two modules are tightly interlaced (more than others) and, in some simpler products/projects, could be considered as a single module.

Typically, the engineering module is used to find implementable solutions for given costs and engineering constraints. There are two types of engineering design problems; one in which the design 
variable itself is uncertain (due to manufacturing problems) and another a design has to be made but consequences of the design during the rest of the life time of the product is uncertain which could include both cost uncertainties and production technology uncertainties. In addition, the economic module may provide demands and their uncertainties and hence the engineering module has to consider also these before a solution can be found. The output of the engineering module is both mean costs and (co)variances of costs; it is possible also that these may relate to multiple objectives so costs doesn't mean only money units. It could be others like time, pollution level, etc.

The REPUSA problem can be used for explaining some of the issues such as the design variable uncertainty versus design under uncertainty in consequences. Photovoltaic (PV) wafers are manufactured in large quantities in integrated manufacturing. Depending upon the material and processes used, the performance (due to components in the wafer) of the PV cells are uncertain, for now, consider just the light to energy conversion efficiency which is the main reason for using PV cells. Therefore, the total energy produced even under constant light input vary. The better the processes and materials used the less the variability in energy produced but higher the manufacturing cost. Therefore, one of the design problem here is the amount of material/process variability that should be chosen. This is one kind of design problem where design variable is uncertain [14]

A second of problem is how many of these PV cells to install (how many panels in reality) given the energy demand and the uncertainty in energy demand. In addition, there is also input uncertainty (that is the amount of usable light available to convert to energy) and given all these uncertainties each decision we take to install (which cannot be changed in short to medium terms) the energy production (consequence) is uncertain [15]. Engineering module is typically coordinating with the economic module to come up with various decisions and their consequences.

Because manufacturing includes materials and processes it also produces output that are of interest to the environmental module (should we remove $\mathrm{CO}_{2}$ locally [16] or just send it out the atmosphere with a chimney). We already saw that engineering/economics solution also involves national and international issues such as employment versus cheap product cost interacting with the society module. Therefore, we need a method such as SEEEF to be able to elicit various solutions and their impacts on all of society and environment in order to make a decision. The decision itself now depends on value systems elicited by the societal module interacting with all stakeholders.

\section{Social-Environmental-Economical-Engineering-based-design Framework (SEEEF)}

Design for society and sustainable design has been studied at least since the early 1990s [17-20] and new organizations have been started by various private, public groups and governmental organizations. The awareness and working towards sustainability is very high but tools for practical implementations is lacking, a common mistake of many of these works is to ignore the engineers while the even more common mistake of engineers has been not getting enough training and inputs in society and environment [21]. After all, at the end, most implementations require engineering and, if engineers have not been part of this movement and have been trained to think in the same holistic way, there is little chance for real change. One of the earliest institution to encourage engineers to think holistically is [22] and even that program requires much change as sustainability is not the central theme of the program. A common fear of asking for sustainability in design is that it is not practical for use today. What was lacking in our designs and policies is that while engineers solved the society's requirement for a project or product with the knowledge and skills available at that time, there was no systematic analysis of what parts of their solution should be only be used for limited time requiring changes or equivalent replacements for an improved sustainability (an example is the replacement of CFCs from cooling appliances, although the science connecting CFC to ozone hole came late and a knowledge that would have been unavailable during the design period; hence the need for continuous assessment and encouragement of independent and fundamental scientific 
research; on the other hand the use of microbeads in consumer products could have been easily prevented from being used at design stage if some care in training of designers in sustainability had taken place and design for sustainability had been encouraged). The major theme of SEEEF is that any product considered in design will have list of issues regarding their suitability to meet societal/environmental constraints identified and plans made over identified length of time to change to meet these constraints. We have started doing these in patch works of problems, for example, when new pipes are laid in water system, most regulations today require us to have no lead in new pipes (as lead has been found to be harmful to people and environment); reducing and realigning roads to encourage less motor vehicles and reduce speeding, and more bicycling, etc. is another example. On the other hand, SEEEF can be used now to redesign existing products as well as new products and projects.

\section{Final Remarks}

Since the 1990s designing for society and environment has been promoted and many tools that can be applied with focus on some specific issues have been coming up. Some of them like donut economics is comprehensive but are not specifically design tools and some like environmental life cycle based designs are good but not comprehensive, for example, they do not consider serious social issues like employment and trade. SEEEF is promoted to overcome these shortcomings and provide for a true interdisciplinary frame work where specialists from humanities, and engineering/science take appropriate roles. SEEEF forces communication with stakeholders and among all specialists an essential part of the decision making process to achieve either product redesigns or new designs of products and projects.

Author Contributions: All authors have been discussing and publishing papers with design issues together for over two decades. KP wrote the first draft of this paper; AS did review of the material and JM reviewed further.

Funding: Author KP thanks NSERC Discovery Grant "Multidisciplinary design optimization under uncertainty" during 2015-20 and the "Design optimization under uncertainty" grant before that.

Acknowledgments: Author KP has been part of the Department of Systems Design Engineering, University of Waterloo for over 30 years of its 50 years' life. Much of his systems thinking and design methodologies were influenced by faculty and students of the department.

Conflicts of Interest: The authors declare no conflict of interest.

\section{References}

1. Ponnambalam, K.; Mousavi, S.J. CHNS Modeling for Study and Management of Human-Water Interactions at Multiple Scales. Water 2020, 12, 1699.

2. Mestre A; Cooper, T. Circular Product Design. A Multiple Loops Life Cycle Design Approach for the Circular Economy, The Design Journal 2017 DOI: 10.1080/14606925.2017.1352686

3. Sakao, T; Neramballi, A. A Product/Service System Design Schema: Application to Big Data Analytics, Sustainability 2020, 12, 3484; doi:10.3390/su12083484

4. Jafar, I., et al. Product Design for Sustainability: A New Assessment Methodology and Case Studies, In book: Environmentally Conscious Mechanical Design 2007, DOI: 10.1002/9780470168202.ch2

5. Zhang, H; Nagel, J.K; Al-Qas, A; Gibbons, E; Lee, J.J; Additive Manufacturing with Bioinspired Sustainable Product Design: A Conceptual Model, Procedia Manufacturing, 2018, 26, 880-891, DOI: 10.1016/j.promfg.2018.07.113

6. Bhamra,T; Lilley, D; Tang, T. Design for Sustainable Behaviour: Using Products to Change Consumer Behaviour, The Design Journal 2018 14:4, 427-445, DOI: 10.2752/175630611X13091688930453

7. https://www.theguardian.com/world/2020/apr/08/amsterdam-doughnut-model-mend-postcoronavirus-economy

8. https://www.kateraworth.com/doughnut/

9. https://www.theguardian.com/us-news/2020/jul/14/joe-biden-climate-jobs-plan 
10. https://www.setac.org/page/IGLCA?\&hhsearchterms=\%221ca $\% 22$

11. Saaty, R.W. The analytic hierarchy process - what it is and how it is used, Mathematical Modelling, 1987, 9, 161-176.

12. Green, P.E.; Srinivasan, V. Conjoint Analysis in Consumer Research: Issues and Outlook, Journal of Consumer Research,1975, 5 (2), 103-238, https://dx.doi.org/10.1086/208721

13. http://www.OpenLCA.org

14. Seifi, A.; Ponnambalam, K; Vlach, J. Optimization of filter designs with dependent and asymmetrically distributed parameters, Journal of Franklin Institute 2013, 350(2), 378-396.

15. Narayan, A; Ponnambalam, K. Risk-averse stochastic programming approach for microgrid planning under uncertainty, Renewable Energy 2017, 101, 399-408.

16. Mukherjee, S; Kumar,P; Yang, A; Fennell, P. Energy and exergy analysis of chemical looping combustion technology and comparison with pre-combustion and oxy-fuel combustion technologies for CO2 capture, Journal of environmental chemical engineering, 2015, 3(3), 2104-2114

17. Whiteley, N; Design for society, Reaktion Books; Revised ed. Edition, 1997

18. Tromp, N; Hekkert, P; Designing for society: Products and Services for a Better World, Bloomsbury publ., 2018.

19. https://www.designsociety.org/

20. Egenhoefer, R.B. Handbook of Sustainable Design, 2018, Routledge Publ.

21. Govindaraj, T. Social and environmental perspectives in the design of engineering and service systems, Int. J. Engng Ed., 2003, 19 (1) 16-24.

22. https://uwaterloo.ca/systems-design-engineering/ 